\title{
THE NSP2 PROTEINS OF MOUSE HEPATITIS VIRUS AND SARS CORONAVIRUS ARE DISPENSABLE FOR VIRAL REPLICATION
}

\author{
Rachel L. Graham, Amy C. Sims, Ralph S. Baric, and Mark R. Denison*
}

\section{INTRODUCTION}

The positive-strand RNA genome of the coronaviruses is translated from ORF1 to yield polyproteins that are proteolytically processed into intermediate and mature nonstructural proteins (nsps). Murine hepatitis virus (MHV) and severe acute respiratory syndrome coronavirus (SARS-CoV) polyproteins incorporate 16 protein domains (nsps), with nsp1 and nsp2 being the most variable among the coronaviruses and having no experimentally confirmed or predicted functions in replication. To determine if nsp2 is essential for viral replication, MHV and SARS-CoV genome RNA was generated with deletions of the nsp2 coding sequence (MHV $\Delta \mathrm{nsp} 2$ and SARS $\Delta \mathrm{nsp} 2$ ). Infectious MHV $\Delta$ nsp2 and SARS $\Delta$ nsp2 viruses were recovered from electroporated cells. The $\Delta \mathrm{nsp} 2$ mutant viruses lacked expression of both nsp2 and an nsp2-nsp3 precursor, but cleaved the engineered chimeric nsp $1 / 3$ cleavage site as efficiently as the native nsp1nsp2 cleavage site. Replication complexes in MHVDnsp2-infected cells lacked nsp2 but were morphologically indistinguishable from those of wild-type MHV by immunofluorescence. These results demonstrate that while nsp2 of MHV and SARS-CoV is dispensable for viral replication in cell culture, deletion of the nsp2 coding sequence attenuates viral growth and RNA synthesis. These findings also provide a system for the study of determinants of nsp targeting and function.

\section{MATERIALS AND METHODS}

\subsection{Generation of Recombinant Viruses}

Deletions of the nsp2 coding sequence were engineered into the infectious cDNAs of MHV and SARS-CoV by deleting nt 951-2705 (aa Val248-832Ala) for MHV and nt

* Vanderbilt University, Nashville, Tennessee 37232. 
805-2718 (aa Ala181-818Gly) for SARS-CoV. Mutant viruses were then generated as previously described. ${ }^{1,2}$

\subsection{Virus Infection, Immunoprecipitation, and Immunoblot}

DBT cells were infected with MHV at an MOI of 5 pfu/cell. At 4.5 h p.i., cells were incubated in medium lacking methionine and cysteine and supplemented with Actinomycin D. $\left[{ }^{35} \mathrm{~S}\right]$-methionine/cysteine was added at $6 \mathrm{~h}$ p.i., and cells were then harvested at $10 \mathrm{~h}$ p.i. Lysates were then immunoprecipitated using antibodies against nsp1, nsp2, nsp3, and nsp8. Proteins were then resolved and visualized by SDS-PAGE and fluorography.

Vero-E6 cells were infected with SARS-CoV at an MOI of 1 PFU/cell. At $12 \mathrm{~h}$ p.i., cells were harvested and lysates resolved by SDS-PAGE. Separated proteins were transferred to nitrocellulose, and nsp1, nsp2, nsp3, and nsp8 antibodies were used to detect viral proteins as described. ${ }^{3}$

\subsection{Immunofluorescence}

DBT cells were grown on glass coverslips as previously described. ${ }^{4}$ Cells were then infected for $6.5 \mathrm{~h}$, fixed and permeablized in $-20^{\circ} \mathrm{C}$ methanol, and processed for immunofluorescence as described. ${ }^{5}$

\subsection{Confocal Microscopy}

All cell images were acquired on a Zeiss 510 LSM laser scanning confocal microscope. Images were acquired using lasers at $488 \mathrm{~nm}$ (green) and $543 \mathrm{~nm}$ (red) and using a 40X, 1.3 NA oil immersion objective. Images were processed using Adobe Photoshop CS.

\section{RESULTS AND DISCUSSION}

\subsection{Deletion of Nsp2 Demonstrates Polyprotein Tolerance to Change}

The deletion of the nsp2 coding sequence from both MHV and SARS-CoV yielded infectious virus. Retention of the engineered deletion was confirmed by sequencing of viral RNA from progeny viruses as well as by detection of viral proteins from infected cells by immunoprecipitation (MHV, Fig. 1) and immunoblot (SARS-CoV, data not shown). No additional mutations were noted in the regions sequenced, consisting of bases $\sim 500 \mathrm{nt} 5^{\prime}$ ' and 3' of the deletion for MHV and $\sim 250 \mathrm{nt} 5^{\prime}$ and 3' of the deletion for SARS-CoV.

Deletion of the nsp2 coding region of these two viruses is, to our knowledge, the first demonstration of the deletion of the coding region of an entire protein domain from a RNA virus polyprotein. This deletion illustrates a previously unrealized flexibility within the coronavirus polyprotein. 


\subsection{Deletion of Nsp2 Does Not Interfere with Polyprotein Processing}

To determine if the deletion of the nsp2 coding region altered processing within the replicase polyprotein, MHV-infected cells were radiolabeled with $\left[{ }^{35} \mathrm{~S}\right]$-methionine/ cysteine, lysed, and viral proteins were detected by immunoprecipitation (Fig. 1). While MHVAnsp2 mutant virus produced no detectable nsp2 protein, all other tested proteins (nsp1, nsp3, and nsp8) were processed comparably to wild type, demonstrating that deletion of nsp2 from the viral polyprotein did not inhibit polyprotein translation or processing. Notably, infection with the MHVAnsp2 virus did not produce the high molecular weight $(\sim 275 \mathrm{kDa})$ protein that has been tentatively identified in previous studies as the nsp2-3 precursor protein. ${ }^{6,7}$ Similarly, lysates generated from SARS-CoV infected cells were probed for nsp1, nsp2, nsp3, and nsp8 proteins by immunoblot (data not shown). As with MHV, the SARS-CoVAnsp2 virus did not produce detectable nsp2 protein, though nsp1, nsp3, and nsp8 were all expressed and processed comparably to wild type.

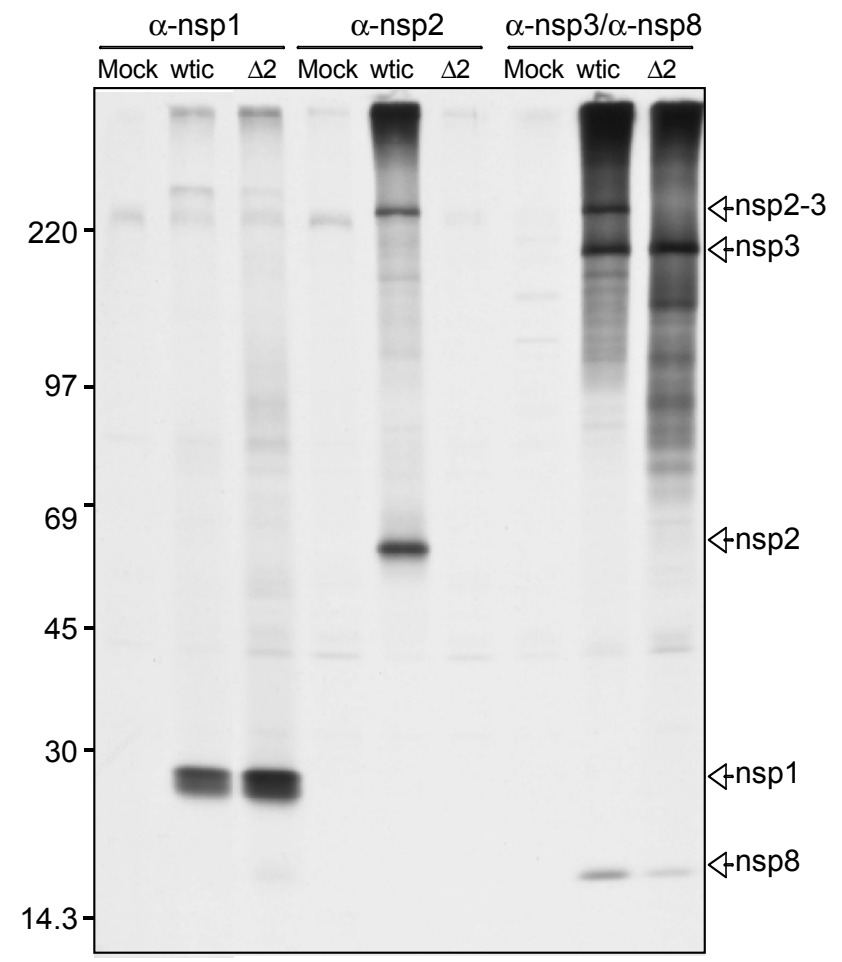

Figure 1. Expression and processing in MHVAnsp2 mutant. DBT cells were mock-infected, infected with recombinant wild-type MHV (wtic), or MHV $\Delta \mathrm{nsp} 2(\Delta 2)$, radiolabeled with $\left[{ }^{35} \mathrm{~S}\right]$-methionine/cysteine, lysed, and immunoprecipitated with antibodies against the indicated proteins. Proteins were then resolved by SDSPAGE and imaged by fluorography. 


\subsection{Replication Complexes Form in the Absence of Nsp2}

To determine if replication complexes formed in cells infected with the MHV $\Delta \mathrm{nsp} 2$ mutant virus, DBT cells were infected with either wild-type or MHVAnsp2 for $6.5 \mathrm{~h}$, fixed, and stained by indirect immunofluorescence for the viral proteins nsp2, nsp8, N (nucleocapsid), which localize to punctate cytoplasmic foci (replication complexes), and M, a marker for sites of viral assembly (Fig. 2). Cells infected with MHV $\Delta$ nsp2 viruses produced no detectable nsp2. However, nsp8 and $\mathrm{N}$ proteins both localized to punctate cytoplasmic focal patterns indistinguishable from wild-type viral replication complexes as well as colocalized with each other. This staining pattern was distinct from the staining pattern of the viral $M$ protein. These results suggest that the MHVAnsp2 virus was capable of forming replication complexes in the absence of the nsp2 protein.

\section{SUMMARY}

The results presented here demonstrate that the MHV and SARS-CoV nsp2 proteins are not required for the production of infectious virus, for polyprotein expression or processing, or for viral replication complex formation in cell culture. The nsp2 protein

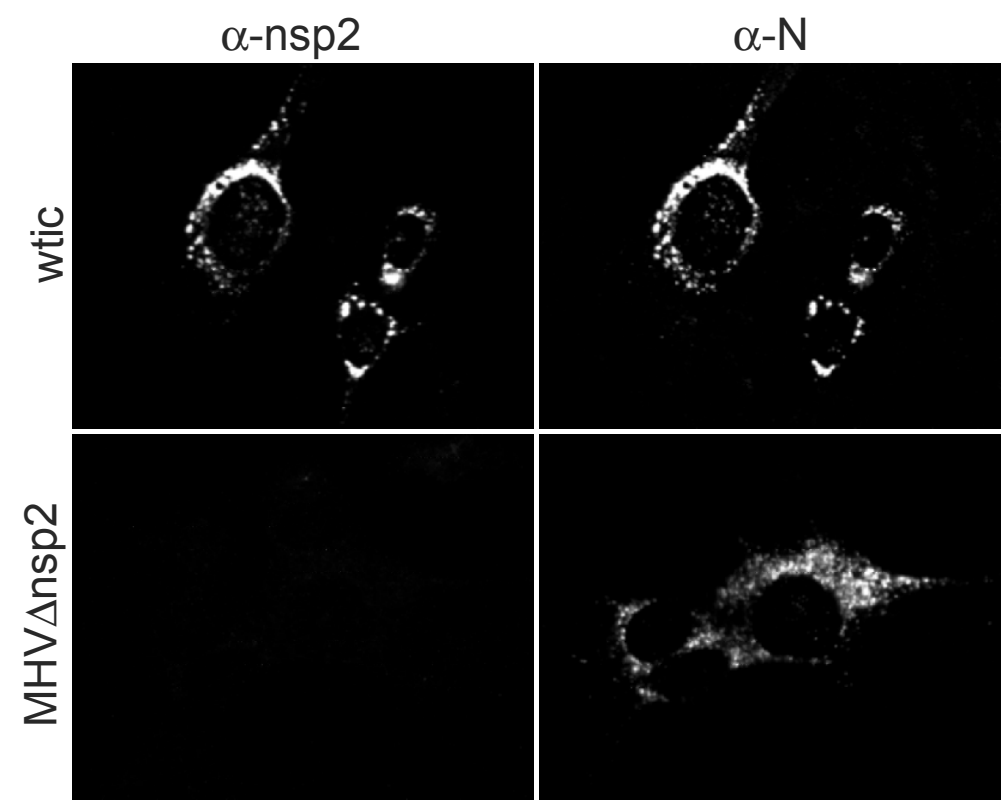

Figure 2. Replication complex formation in MHVAnsp2 mutant. DBT cells on glass coverslips were infected with either recombinant wild-type MHV (wtic) or MHV $\Delta \mathrm{nsp} 2$ for $6.5 \mathrm{~h}$, then fixed and permeablized in $-20^{\circ} \mathrm{C}$ methanol. Cells were then stained using antibodies against nsp2 and nucleocapsid protein $(\mathrm{N})$, a marker for replication complexes. 
domain resides in a region of the coronavirus replicase that is relatively nonconserved across coronaviruses. In fact, the size and amino acid sequence variability of nsp 2 across the different coronaviruses has led some investigators to speculate that the nsp 2 protein, along with the nsp1 and nsp3 proteins, may play host- and/or cell-specific roles in the virus life cycle. ${ }^{8-10}$ While this may be the case, it should be noted that nsp2, in some form, exists in all coronaviruses studied to date and likely plays a pivotal role in the viral life cycle. A previous study from our laboratory identified a coronavirus replicase protein that plays an important role in viral pathogenesis. ${ }^{11}$ Such may prove to be the case for nsp2, as well. Alternatively, beacuse nsp2 exists as a detectable precursor protein nsp2-3 prior to processing of nsp2 and nsp3 into mature proteins, nsp2 may play a critical adaptor/regulatory role for nsp3 function. Importantly, the viruses produced in this study provide a system by which the role of the nsp 2 protein in viral infection can be characterized.

\section{ACKNOWLEDGMENTS}

This work was supported by National Institutes of Health grants RO1 AI26603 (M.R.D.), RO1 AI059136-01 (R.S.B.), and PO1 AI059443-01 (R.S.B. and M.R.D.) and the Training Grant for Cellular, Biochemical, and Molecular Sciences, Vanderbilt University School of Medicine 5T32GM00855 (R.L.G.). Additional support was provided by the Public Health Service award CA68485 for the Vanderbilt DNA Sequencing Shared Resource and the Molecular Imaging Shared Resource of the Vanderbilt-Ingram Cancer Center.

\section{REFERENCES}

1. Yount, B., Denison, M. R., Weiss, S. R., and Baric, R. S., 2002, Systematic assembly of a full-length infectious cDNA of mouse hepatitis virus strain A59, J. Virol. 76, 11065-11078.

2. Yount, B., Curtis, K. M., Fritz, E. A., Hensley, L. E., Jahrling, P. B., Prentice, E., Denison, M. R., Geisbert, T. W., and Baric, R. S., 2003, Reverse genetics with a full-length infectious cDNA of severe acute respiratory syndrome coronavirus, Proc. Natl. Acad. Sci. USA 100, 12995-13000.

3. Prentice, E., McAuliffe, J., Lu, X., Subbarao, K., and Denison, M. R., 2004, Identification and characterization of severe acute respiratory syndrome coronavirus replicase proteins, J. Virol. 78, $9977-$ 9986.

4. Bost, A. G., Carnahan, R. H., Lu, X. T., and Denison, M. R., 2000, Four proteins processed from the replicase gene polyprotein of mouse hepatitis virus colocalize in the cell periphery and adjacent to sites of virion assembly, J. Virol. 74, 3379-3387.

5. Denison, M. R., Spaan, J. M., van der Meer, Y., Gibson, C. A., Sims, A. C., Prentice, E., and Lu, X. T, 1999, The putative helicase of the coronavirus mouse hepatitis virus is processed from the replicase gene polyprotein and localizes in complexes that are active in viral RNA synthesis, J. Virol. 73, 6862-6871.

6. Denison, M. R., Hughes, S. A., and Weiss, S. R., 1995, Identification and characterization of a 65-kDa protein processed from the gene 1 polyprotein of the murine coronavirus MHV-A59, Virology 207, 316-320.

7. Schiller, J. J., Kanjanahaluethai, A., and Baker, S. C., 1998, Processing of the coronavirus mhv-jhm polymerase polyprotein: identification of precursors and proteolytic products spanning 400 kilodaltons of ORF1a, Virology 242, 288-302.

8. de Vries, A. A. F., Horzinek, M. C., Rottier, P. J. M., and deGroot, R. J., 1997, The genome organization of the nidovirales: similarities and differenced between arteri-, toro, and coronaviruses, Sem. Virology 8 33-47.

9. Ziebuhr, J., Snijder, E. J., and Gorbalenya, A. E., 2000, Virus-encoded proteinases and proteolytic processing in the Nidovirales, J. Gen. Virol. 81, 853-879. 
10. Ziebuhr, J., Thiel, V., and Gorbalenya, A. E., 2001, The autocatalytic release of a putative RNA virus transcription factor from its polyprotein precursor involves two paralogous papain-like proteases that cleave the same peptide bond, J. Biol. Chem. 276, 33220-33232.

11. Sperry, S. M., Kazi, L., Graham, R. L., Baric, R. S., Weiss, S. R., and Denison, M. R., 2005, Single-aminoacid substitutions in open reading frame (ORF) 1 b-nsp14 and ORF 2a proteins of the coronavirus mouse hepatitis virus are attenuating in mice, J. Virol. 79, 3391-4000. 